\title{
Phase transitions of biological phenotypes by means of a prototypical PDE model
}

\author{
C. Mascia $^{1 *}$, P. Moschetta ${ }^{1}$, C. Simeoni ${ }^{2}$ \\ ${ }^{1}$ Department of Mathematics G. Castelnuovo, Sapienza University of Rome, Italy \\ ${ }^{2}$ Laboratoire J.A. Dieudonné, University of Nice Sophia Antipolis, France \\ *Email address for correspondence: corrado.mascia@uniroma1.it \\ Communicated by Roberto Natalini \\ Received on 12 09, 2018. Accepted on 11 13, 2019.
}

\begin{abstract}
The basic investigation is the existence and the (numerical) observability of propagating fronts in the framework of the so-called Epithelial-to-Mesenchymal Transition and its reverse Mesenchymal-to-Epithelial Transition, which are known to play a crucial role in tumor development. To this aim, we propose a simplified one-dimensional hyperbolic-parabolic PDE model composed of two equations, one for the representative of the epithelial phenotype, and the second describing the mesenchymal phenotype. The system involves two positive constants, the relaxation time and a measure of invasiveness, moreover an essential feature is the presence of a nonlinear reaction function, typically assumed to be $S$-shaped. An identity characterizing the speed of propagation of the fronts is proven, together with numerical evidence of the existence of traveling waves. The latter is obtained by discretizing the system by means of an implicit-explicit finite difference scheme, then the algorithm is validated by checking the capability of the so-called LeVeque-Yee formula to reproduce the value of the speed furnished by the above cited identity. Once such justification has been achieved, we concentrate on numerical experiments relative to Riemann initial data connecting two stable stationary states of the underlying ODE model. In particular, we detect an explicit transition threshold separating regression regimes from invasive ones, which depends on critical values of the invasiveness parameter. Finally, we perform an extensive sensitivity analysis with respect to the system parameters, exhibiting a subtle dependence for those close to the threshold values, and we postulate some conjectures on the propagating fronts.
\end{abstract}

Keywords: phase transitions, reaction-diffusion systems, propagating fronts, finite difference schemes, wave speed approximations

AMS subject classification: 34C37, 35A24, 35C07, 35K57, 35Q92, 65M06, 92C42

\section{Introduction}

Epithelial-to-Mesenchymal Transition (EMT) and its reverse process Mesenchymal-to-Epithelial Transition (MET) are crucial steps during tissues and organs remodeling, a phenomenon at the basis of what is generally called morphogenesis. Epithelial and mesenchymal cells exhibit different phenotypes, the former being characterized by tight junctions and cohesion, while the latter being more scattered and with a high degree of motility [1,2]. As such, their emergences are also recognized as critical events which enable/forbid cancer cells in acquiring/losing malignant features.

Altogether, EMT and MET display dynamical behaviors which resemble those observed in physical systems during abrupt macroscopic changes between qualitatively separated stable states, also known as phase transitions [3]. A characteristic element to be taken into consideration is that even gradual variation in a few control parameters and/or unknowns density can switch cells into specific and distinct phenotypes.

The description of the interplay between the two phenotypes, namely epithelial and mesenchymal, has been largely explored using various strategies and tools (see [4,5] and references therein). The most traditional approach is based on a bottom-up procedure, supposed to be paramount for describing how global structures are the result of underlying microscopic counterparts. However, in the last decades, such point of view has been widely disputed, leaving the space to different approaches based on special types of modeling programmes [6]. Indeed, it has been proposed that critical events are the result of emerging 
properties at a scale that is larger than the microscopic ones, according to the influence of external constraints. Therefore, a different approach should be applied, based on what it is now a well-established discipline, following the Systems Biology perspective [7]. More specifically, instead of focusing on the role of individual genes, proteins or other local pathways in biological phenomena, its aim is to characterize the ways molecular parts adopt for interacting with each other to determine the collective dynamics of the system as a whole. Recently, the Systems Biology approach has been successfully extended to the field of oncology, with the ambitious idea of grasping complexity in the genesis of cancer [8].

In this article, we propose a simplified mathematical model which is, in principle, capable to catch the basic qualitative behavior of EMT and MET, in an uncomplicated setting. We stress that the presence of a (single) nonlinear term is crucial, since it guarantees the existence of propagating fronts through the emergence of several stationary states. Incidentally, we observe that the same kind of mechanism can be considered for the description of wound healing experiments [9].

Entering the heart of the matter, we focus here on a diffusive variation of the ODE model originally proposed in [7] which, after an appropriate adimensionalisation, reads as

$$
\left\{\begin{aligned}
\tau \partial_{t} u & =v-u \\
\partial_{t} v & =\Delta_{\mathbf{x}} v-v+\lambda g(u) \quad \text { for } \quad \mathbf{x} \in \mathbb{R}^{d}, \quad t>0,
\end{aligned}\right.
$$

with some positive parameters $\tau, \lambda$ and a saturating-type reaction function $g$, which is typically assumed to be $S$-shaped (more precise assumptions will be stated later on). Roughly speaking, the variable $u$ can be interpreted as the density of cells exhibiting an epithelial phenotype, while the variable $v$ is relative to the mesenchymal phenotype, its motility being described by the presence of the Laplace operator $\Delta_{\mathbf{x}}$ in the second equation ${ }^{\mathrm{a}}$. The main advantage of system (1) is to collect into two constants, namely $\tau$ and $\lambda$, the global characteristics of the physical model: the first corresponds to a relaxation time and the latter describes invasiveness of the tumor cells. On that account, such a model has no intention of providing a quantitative biological description, but only a qualitative one; the main point is to show that the invasion process could be, in principle, reversible and that such reversion could be measured by means of a limited number of parameters. A realistic quantitative model would require a more tailored definition of the objects under examination.

The manuscript is structured as follows. In Section 2, we present the general form of the mathematical model and deduce its corresponding adimensionalisation [7]. After a brief discussion on the basic properties of the underlying kinetic mechanism, that is relative to the description of space-independent solutions, we consider the special case of planar solutions, then concentrating the attention to traveling wave solutions for which an identity for the propagation speed of the front is determined. Section 3 concerns the computational analysis of the one-dimensional version of the model, choosing an implicit-explicit finite difference algorithm (the linear terms are discretized implicitly and an explicit approximation is reserved to the nonlinear term). Several numerical simulations are validated by considering the so-called LeVeque-Yee formula as a reliability reference for the evaluation of propagation speed. In addition, we perform a sensitivity analysis with respect to the parameters $\tau$ and $\lambda$, collecting the conclusions of the analysis performed throughout this article.

\section{A simple PDE model for phase transitions}

Let $\Omega$ denote a domain in $\mathbb{R}^{d}$ with smooth boundary $\partial \Omega$. We consider the initial-boundary value problem for the system of partial differential equations

$$
\left\{\begin{array}{l}
\tau \partial_{t} u=\alpha v-\beta u \\
\gamma \partial_{t} v=\varepsilon^{2} \Delta_{\mathbf{x}} v-\mu v+\lambda g(u) \quad \text { for } \quad \mathbf{x} \in \Omega, \quad t>0,
\end{array}\right.
$$

\footnotetext{
${ }^{a}$ The interpretation of the unknowns provided in [7] is actually different: the variable $u$ refers to the E-cadherin boundary values and $v$ stands for the coherency. Being the latter too vague to be rigoroursly quantified, here we have opted for an alternative meaning in terms of cells phenotypes, which appear to be experimentally more robust and, ideally, measurable.
} 
with some external parameters $\tau, \alpha, \beta, \gamma, \varepsilon, \lambda, \mu$ and a structural function $g$ to be specified later on. This system is determined by the (non-negative) initial conditions

$$
u(\mathbf{x}, 0)=u_{0}(\mathbf{x}), \quad v(\mathbf{x}, 0)=v_{0}(\mathbf{x}),
$$

and the zero-flux boundary conditions

$$
\left.\nabla_{\mathbf{x}} u \cdot \mathbf{n}\right|_{\partial \Omega}=0,\left.\quad \nabla_{\mathbf{x}} v \cdot \mathbf{n}\right|_{\partial \Omega}=0
$$

where $\mathbf{n}$ denotes the exterior normal vector of the boundary $\partial \Omega$. We remark that assumption (4) is biologically meaningful especially when dealing with in vitro experiments settled on a Petri dish, for example. However, for the mathematical analysis, the requirement on boundary data for the unknown $u$ is not needed, because its dynamics is described by an ODE model.

The reaction function $g$ in (2) is assumed to be a transformation of $[0,+\infty)$ into itself and, additionally, it satisfies the following hypotheses:

H1. it is sufficiently smooth, strictly increasing and such that $g(0)=0$;

H2. it is convex in $[0, \bar{u}]$ and concave in $[\bar{u},+\infty)$ for some $\bar{u}>0$;

H3. it converges toward a given positive (saturating) limit $g_{\infty}$ at $+\infty$.

\subsection{Adimensionalization}

We apply to system (2) the rescaling

$$
\tilde{u}=\frac{u}{U}, \quad \tilde{v}=\frac{v}{V}, \quad s=\frac{t}{T}, \quad \mathbf{y}=\frac{\mathbf{x}}{X},
$$

so that it becomes

$$
\left\{\begin{aligned}
\tilde{\tau} \partial_{s} \tilde{u} & =\tilde{v}-\tilde{u} \\
\partial_{s} \tilde{v} & =\Delta_{\mathbf{y}} \tilde{v}-\tilde{v}+\tilde{\lambda} \tilde{g}(\tilde{u})
\end{aligned}\right.
$$

where

$$
V=\frac{\beta U}{\alpha}, \quad T=\frac{\gamma}{\mu}, \quad X=\frac{\varepsilon}{\sqrt{\mu}} \quad \text { and } \quad \tilde{\tau}=\frac{\mu \tau}{\beta \gamma}, \quad \tilde{\lambda}=\frac{\alpha \lambda}{\beta \mu},
$$

together with $\tilde{g}(\tilde{u}):=\frac{1}{U} g(U \tilde{u})$, and choosing the constant value $U$ for $\tilde{g}$ to be equal to 1 at $+\infty$, that is

$$
U=g_{\infty}:=g(+\infty)
$$

Hence, from now on, we study the solutions to the adimensionalized hyperbolic-parabolic system (1), where, for the sake of readability, we consider the original variables $(\mathbf{x}, t)$, the parameters $\tau, \lambda$ and unknowns $(u, v)$, also assuming $g(+\infty)=1$, with the initial-boundary conditions described in (3)-(4).

It is worth noticing that first equation in (1) can also be rewritten as a truncated first order Taylor expansion of the delayed expression

$$
u(\mathbf{x}, t+\tau)=u(\mathbf{x}, t)+\tau \partial_{t} u(\mathbf{x}, t)+o(\tau) \approx u(\mathbf{x}, t)+\tau \partial_{t} u(\mathbf{x}, t) .
$$

Moreover, system (1) is equivalent to the one-field equation for the unknown $u$ given by

$$
\tau \partial_{t t} u+(1+\tau) \partial_{t} u=\Delta_{\mathbf{x}}\left(u+\tau \partial_{t} u\right)-u+\lambda g(u)
$$

which is a third order hyperbolic equation for the scalar variable $u$. Viceversa, the system (1) is also equivalent to an integro-differential parabolic equation for the unknown $v$ given by

$$
\partial_{t} v=\Delta_{\mathbf{x}} v-v+\lambda g\left(u_{0}(\mathbf{x}) e^{-t / \tau}+\frac{1}{\tau} \int_{0}^{t} e^{-(t-s) / \tau} v(\mathbf{x}, s) d s\right)
$$


which is obtained by solving the first equation in (1) with respect to $u$ and then coupling with the second condition for the variable $v$.

Incidentally, let us observe that system (1) is positivity preserving, meaning that if the initial data $u_{0}$ and $v_{0}$ are non-negative, the same is true for the solutions to the corresponding initial-boundary value problem. Indeed, the two equations of the system, considered separately, are scalar equations; thus, they both satisfy a comparison principle: in particular, the solution $u$ to the linear ODE model

$$
\tau \partial_{t} u+u=F(\mathbf{x}, t) \geq 0, \quad u(\mathbf{x}, 0)=u_{0}(\mathbf{x}) \geq 0,
$$

is non-negative, and similarly the solution $v$ to the linear parabolic operator

$$
\partial_{t} v-\Delta_{\mathbf{x}} v+v=G(\mathbf{x}, t) \geq 0, \quad v(\mathbf{x}, 0)=v_{0}(\mathbf{x}) \geq 0,
$$

is non-negative (with both conditions being satisfied by the model under analysis).

\subsection{Space independent solutions}

To start with, let us consider space independent solutions, so that system (1) reduces to

$$
\tau \frac{d u}{d t}=v-u, \quad \frac{d v}{d t}=-v+\lambda g(u)
$$

which coincides with the ODE model originally proposed in [7]. Analogous models are already present in the literature since decades: among others, we quote [10] and its descendants, where the FitzHugh-Nagumo system is proposed in the context of axon signalling, with variables $u$ and $v$ describing approximately the potential of the nerve axons and a (qualitative) feature of the ionic channels opening/closure mechanism, respectively. Of course, the action of the variable $u$ inside the equation for $v$ is completely different with respect to the model presented in this article: indeed, we attempt at simulating a different type of cellular mechanism, characterized by a cooperative-type coupling, for which each variable positively contributes to the increase of the other. Finally, in [11] a similar system based on the mass action law is considered in the context of wound healing experiments [9], with the variables $u$ and $v$ describing, respectively, the area of dead tissue and the spatially-evolving section of the wound.

The main interest in the model presented in this article is the form of the reaction function $g$, which is supposed to have a saturating Hill shape, and concrete examples are provided by

$$
g(u)=\frac{u^{p}}{1+u^{p}}=1-\frac{1}{1+u^{p}},
$$

with $p \geq 2$. Ideally, the formula (7) can be recovered by applying some variation of the Michaelis-Menten reduction from a more involved enzyme-type model (see [12-14], for instance) and this kind of shape is sometimes also referred to as the Langmuir adsorption model function [15]. Numerical simulations will be provided in Section 3.3 for the special case $p=2$, for which the modeling function $g$ is said to have a Holling type III response form [16].

Next, we concentrate on the steady states of the ODE system (6), which are also steady stationary states of the original PDE system (1). Time independent solutions are couple of real numbers such that $v-u=0$ and $v-\lambda g(u)=0$, hence they correspond to values $\bar{u}$ such that

$$
h(\bar{u} ; \lambda):=\bar{u}-\lambda g(\bar{u})=0 .
$$

Under the additional assumptions on the alternating convexity/concavity of $g$ introduced above (see $\mathbf{H 1}$, $\mathbf{H 2}$ and $\mathbf{H 3}$ at the beginning of Section 2), it is possible to distinguish three different configurations depending on the value taken by the external parameter $\lambda$ (refer to Figure 1).

Specifically, there is a (strictly) positive threshold value $\lambda_{\star}>0$, such that:

1. for $\lambda<\lambda_{\star}$, the point $(0,0)$ is the unique intersection of the straight line $v=u$ with the curve $v=\lambda g(u)$, which corresponds to a stable equilibrium for the underlying ODE system; 


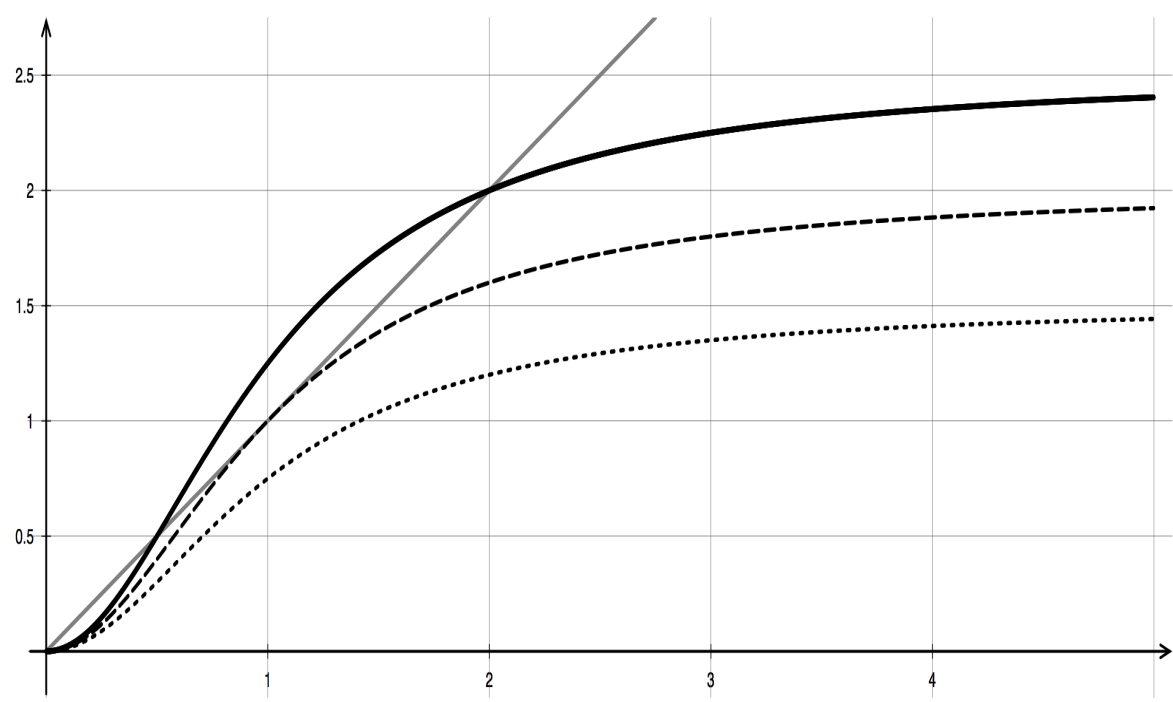

Figure 1. Graphs of the straight line $v=u$ (continuous, gray) and the reaction function $v=\lambda g(u)$, with $g(u)=\frac{u^{2}}{1+u^{2}}$, for $\lambda=1.5$ (dotted), $\lambda=2.0$ (dashed) and $\lambda=2.5$ (continuous).

2. for $\lambda=\lambda_{\star}$, there are two intersections with abscissae $u=0$ and $u=u_{\star}>0$ (unstable state);

3. for $\lambda>\lambda_{\star}$, there are three intersections for $u=0, u=u_{-}$and $u=u_{+}$such that $0<u_{-}<u_{+}$, with the extremal values which correspond to stable equilibria and the intermediate one that is unstable for the underlying ODE system.

The threshold $\lambda_{\star}$, which separates the two limiting regimes, is given by the unique value such that

$$
h\left(u_{\star} ; \lambda_{\star}\right)=u_{\star}-\lambda_{\star} g\left(u_{\star}\right)=0 \quad \text { and } \quad \frac{d h}{d u}\left(u_{\star} ; \lambda_{\star}\right)=1-\lambda_{\star} \frac{d g}{d u}\left(u_{\star}\right)=0,
$$

for some $u_{\star}>0$. Hence, it holds $\lambda_{\star}=\frac{u_{\star}}{g\left(u_{\star}\right)}$ and $\frac{1}{\lambda_{\star}}=\frac{d g}{d u}\left(u_{\star}\right)$, so that finally we obtain the pointwise identity

$$
u_{\star} \frac{d g}{d u}\left(u_{\star}\right)=g\left(u_{\star}\right) .
$$

Example 2.1. We consider an example of reaction function (7) with $p=2$. For this case, the intersection values $\bar{u}$ in (8) are explicitly computable: upon substitution, for $\lambda \geq 2$, we infer that $h(\bar{u} ; \lambda)=0$ if and only if $\bar{u}\left(\bar{u}^{2}-\lambda \bar{u}+1\right)=0$, that is $\bar{u} \in\left\{0, u_{-}(\lambda), u_{+}(\lambda)\right\}$ with

$$
u_{ \pm}(\lambda)=\frac{1}{2}\left(\lambda \pm \sqrt{\lambda^{2}-4}\right) .
$$

For $0<\lambda<2, h(\bar{u} ; \lambda)=0$ for physically admissible solutions if and only if $\bar{u}=0$. In particular, the threshold value is $\lambda_{\star}=2.0$, corresponding to the abscissa $u_{\star}=1$ (refer to Figure 2). As for the general case, the value $u_{+}(\lambda)$ is monotone increasing with respect to the invasiveness parameter $\lambda$, and it holds

$$
u_{+}(\lambda)=\lambda+o(1) \quad \text { as } \quad \lambda \rightarrow+\infty .
$$

\subsection{Stability of steady states}

We start by dealing with the limiting regime $\tau=0$ in system (6). Denoting by $H(\cdot ; \lambda)$ the primitive with respect to the first argument of the function $h(\cdot ; \lambda)$ and by $G$ the primitive of $g$, we deduce that

$$
H(v ; \lambda)=\int_{0}^{v}[u-\lambda g(u)] d u=\frac{1}{2} v^{2}-\lambda G(v),
$$




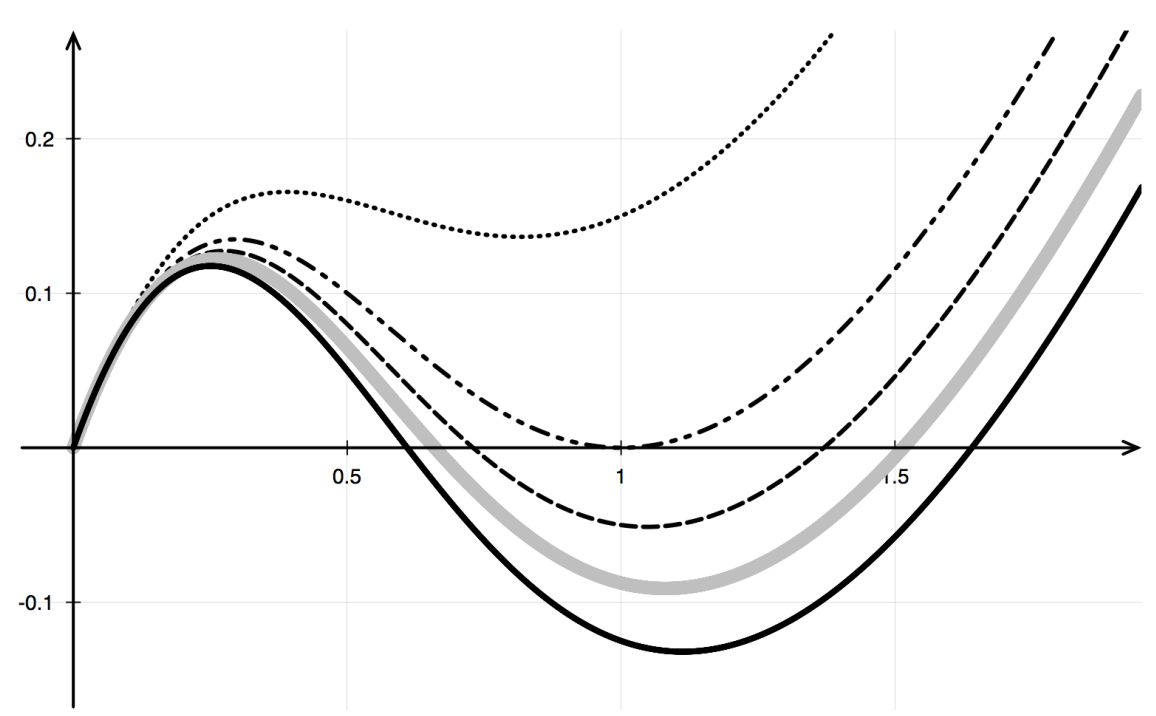

Figure 2. Graphs of the function $h(u ; \lambda)=u-\lambda g(u)$, with $g(u)=\frac{u^{2}}{1+u^{2}}$, for $\lambda=1.7$ (dotted), $\lambda=\lambda_{\star}=2.0($ dot-dashed), $\lambda=2.1$ (dashed), $\lambda=\lambda_{0}=2.175063$ (continuous, gray) and $\lambda=2.25$ (continuous).

which is called the potential of the function $h$ defined in (8), because the dynamical system (6) with $\tau=0$ is equivalent to the scalar equation (ODE in gradient formulation)

$$
\frac{d v}{d t}=-\partial_{v} H(v ; \lambda)
$$

Note that there exists a unique positive $\lambda_{0}$ and its corresponding $u_{0}:=u_{+}\left(\lambda_{0}\right)$ such that the two wells of the potential $H$ have the same depth (refer to Figure 3). For the prototype reaction function (7) with $p=2$, the values $\lambda_{0}$ and $u_{0}$ are explicitly computable by applying the iterative Newton method, so that

$$
u_{0} \approx 1.5149946 \quad \text { and } \quad \lambda_{0} \approx 2.175063>\lambda_{\star}=2.0 \text {. }
$$

Such configuration is particularly relevant, as it will be further discussed later on.

For $\tau=0$, the stability of the equilibria for equation (10) is readily checked, being determined by the sign of the derivative of the function $h(\cdot ; \lambda)$ in (8) with respect to the first argument: when this derivative is positive, the equilibrium is stable, and when negative, the equilibrium is unstable.

For $\tau>0$, the situation is only slightly more complicated. Indeed, the linearization of equation (5) for space independent solutions at some equilibrium $\tilde{u}$ reads

$$
\tau \frac{d^{2} u}{d t^{2}}+(1+\tau) \frac{d u}{d t}+\partial_{u} h(\tilde{u} ; \lambda) u=0
$$

and the corresponding characteristic values are

$$
\mu_{ \pm}(\tau)=\frac{1}{2 \tau}\left[-(1+\tau) \pm \sqrt{(1-\tau)^{2}+4 \tau \lambda \frac{d g}{d u}}\right]
$$

In particular, it is readily checked that $\mu_{-}$is always strictly negative (with order $1 / \tau$ ) and $\mu_{+}$is negative (with order 1) if and only if

$$
\partial_{u} h(\tilde{u} ; \lambda)=1-\lambda \frac{d g}{d u}(\tilde{u})>0,
$$

matching completely the limiting regime $\tau \rightarrow 0^{+}$. 


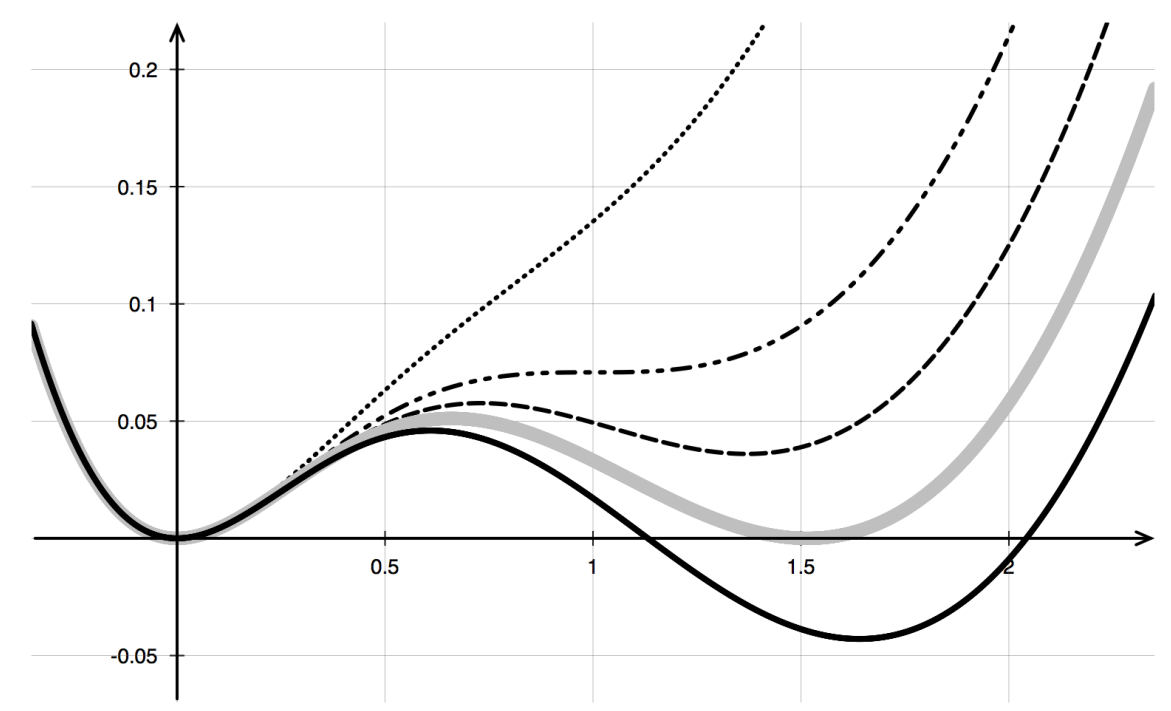

Figure 3. Graphs of the potential $H(u ; \lambda)$ associated with $g(u)=\frac{u^{2}}{1+u^{2}}$ for $\lambda=1.7$ (dotted), $\lambda=\lambda_{\star}=2.0$ (dot-dashed), $\lambda=2.1$ (dashed), $\lambda=\lambda_{0}=2.175063$ (continuous, gray) and $\lambda=2.25$ (continuous).

\subsection{Planar solutions}

Next, let us consider special solutions possessing planar symmetry - labelled as the original unknowns with an abuse of notation - given by $u(\mathbf{k} \cdot \mathbf{x}, t)$ and $v(\mathbf{k} \cdot \mathbf{x}, t)$, for some vector $\mathbf{k} \in \mathbb{R}^{d}$ with unit norm. Substituting this assumption in system (1), we infer the one-dimensional PDE model

$$
\left\{\begin{aligned}
\tau \partial_{t} u & =v-u \\
\partial_{t} v & =\partial_{x x} v-v+\lambda g(u)
\end{aligned}\right.
$$

with the zero-flux boundary conditions (4) reducing to

$$
\partial_{x} v(a, t)=\partial_{x} v(b, t)=0 \quad \text { for } \quad \Omega=(a, b) .
$$

In the case $\Omega=\mathbb{R}$, system (11) is expected to possess special solutions consisting in a rigid motion with some velocity $c$ of a fixed configuration $(U, V)$, according to the following definition.

Definition 2.1. A solution $(u, v)$ of the special form $(u, v)(x, t)=(U, V)(\xi)$, with $\xi:=x-c t$ and asymptotic states $(U, V)( \pm \infty)=\left(U_{ \pm}, V_{ \pm}\right)$with $\left(U_{-}, V_{-}\right) \neq\left(U_{+}, V_{+}\right)$is called a propagating front. The function $(U, V)$ is the profile of the front and $c$ is its speed.

The ODE model for traveling wave solutions deduced from (11) reads

$$
c \tau \frac{d U}{d \xi}+V-U=0, \quad \frac{d^{2} V}{d \xi^{2}}+c \frac{d V}{d \xi}-V+\lambda g(U)=0
$$

In particular, being the system (13) autonomous, the profiles $(U, V)$ - whenever they exist - are determined up to translations in the variable $\xi$ (the so-called translational invariance of traveling fronts). Moreover, it is customarily assumed that $\frac{d V}{d \xi} \rightarrow 0$ as $\xi \rightarrow \pm \infty$, because that is actually the case for solutions with (constant) asymptotic states.

\subsection{An identity for the wave speed}

For $\tau=0$, the existence and stability of a heteroclinic orbit connecting the two stable states is a well known result (see [17] and references therein). Moreover, the corresponding speed is uniquely determined 
by the location of the asymptotic states. In such a singular limiting regime, the model (11) reduces to a "fake" two-dimensional system, being actually equivalent to the scalar reaction-diffusion equation $\partial_{t} v=\partial_{x x} v-\partial_{v} H(v ; \lambda)$, where $H(\cdot ; \lambda)$ is given in $(9)$. Whenever the potential $H$ exhibits two wells, which is the case if and only if $\lambda>\lambda_{\star}$ (refer to Figure 3 ), it can be shown that there exists a propagating front connecting the two minima of $H$, thus corresponding to the extremal zeros of $h$, with speed that is linked to the difference of depth of the wells. Indeed, for $\tau=0$, multiplying the reduced ODE for traveling waves (13) by $\frac{d V}{d \xi}$ and integrating over $\mathbb{R}$, we infer the identity

$$
c(0, \lambda)=\frac{H\left(V_{+} ; \lambda\right)-H\left(V_{-} ; \lambda\right)}{\int_{-\infty}^{+\infty}(d V / d \xi)^{2} d \xi}=\frac{H\left(u_{+} ; \lambda\right)}{\int_{-\infty}^{+\infty}(d V / d \xi)^{2} d \xi} \quad \text { for all } \quad \lambda \geq \lambda_{\star},
$$

where $V_{ \pm}=V( \pm \infty)$ indicate the asymptotic states, with $V_{-}=0$ and $V_{+}=u_{+}$, since $H(0 ; \lambda)=0$.

For $\tau>0$, we can mimic the same procedure obtaining a generalization of the above identity.

Proposition 2.1. Assume that $\tau>0$ is chosen so that system (11) supports traveling wave solutions connecting $(0,0)$ with $\left(u_{+}(\lambda), u_{+}(\lambda)\right)$. Then, the speed of propagation $c(\tau, \lambda)$ satisfies the identity

$$
c(\tau, \lambda)=\frac{H\left(u_{+} ; \lambda\right)}{\int_{-\infty}^{+\infty}\left[\tau \lambda \frac{d g}{d u}(d U / d \xi)^{2}+(d V / d \xi)^{2}\right] d \xi} .
$$

Proof. Multiplying the first equation of system (13) by $\lambda \frac{d g}{d \xi}$ and the second by $\frac{d V}{d \xi}$, we deduce

$$
c \tau \lambda \frac{d g}{d \xi} \frac{d U}{d \xi}+\lambda \frac{d g}{d \xi} V-\lambda \frac{d g}{d \xi} U=0, \quad \frac{d^{2} V}{d \xi^{2}} \frac{d V}{d \xi}+c\left(\frac{d V}{d \xi}\right)^{2}-V \frac{d V}{d \xi}+\lambda g \frac{d V}{d \xi}=0
$$

which can be rewritten as

$$
c \tau \lambda \frac{d g}{d u}\left(\frac{d U}{d \xi}\right)^{2}+\lambda \frac{d g}{d \xi} V-\lambda \frac{d g}{d u} U \frac{d U}{d \xi}=0
$$

and

$$
c\left(\frac{d V}{d \xi}\right)^{2}+\frac{d}{d \xi}\left[\frac{1}{2}\left(\frac{d V}{d \xi}\right)^{2}-\frac{1}{2} V^{2}\right]+\lambda g \frac{d V}{d \xi}=0 .
$$

Then, summing up these two equations and recalling that $G$ is a primitive of $g$, we conclude that

$$
c\left[\tau \lambda \frac{d g}{d u}\left(\frac{d U}{d \xi}\right)^{2}+\left(\frac{d V}{d \xi}\right)^{2}\right]+\frac{d}{d \xi}\left[\frac{1}{2}\left(\frac{d V}{d \xi}\right)^{2}-\frac{1}{2} V^{2}+\lambda G(U)+\lambda(V-U) g(U)\right]=0,
$$

since a straightforward application of the integration by parts formula provides

$$
\int u \frac{d g}{d u} d u=u g(u)-\int g(u) d u=u g(u)-G(u)+\text { constant. }
$$

Finally, integrating with respect to $\xi \in \mathbb{R}$, under the assumption that $\frac{d V}{d \xi} \rightarrow 0$ as $\xi \rightarrow \pm \infty$, we obtain the equality (15) for $U_{-}=V_{-}=0$ and $U_{+}=V_{+}=u_{+}$because $H(0 ; \lambda)=0$ from its definition (9).

It is worth comparing the general identity (15) with its reduced version (14). Moreover, we stress the fact that identity (15) cannot be regarded as an equality, since the right-hand side of the formula depends on the derivatives of the front profile, which in turn depends on the velocity itself. 
There exists a critical value $\lambda_{0}$, strictly greater than $\lambda_{\star}$, such that system (11) possesses a special traveling wave which is actually a stationary solution $(U, V)=(U(x), V(x))$ with null velocity. Such value separates positive and negative speeds of propagation, and it is determined by the requirements

$$
h\left(u_{0} ; \lambda_{0}\right)=u_{0}-\lambda_{0} g\left(u_{0}\right)=0 \quad \text { and } \quad H\left(u_{0} ; \lambda_{0}\right)=\frac{1}{2} u_{0}^{2}-\lambda_{0} G\left(u_{0}\right)=0 .
$$

The first condition above translates the fact that $u_{0}$ is a zero of the function $h(u ; \lambda)$, hence a singular point of the potential $H(u ; \lambda)$, and consequently a candidate for the asymptotic state $u_{+}$in Proposition 2.1. The second one corresponds to the requisite that the two wells of $H$ have same depth (refer to Figure 3 ) and implies that $c\left(\tau, \lambda_{0}\right)=0$ from identity (15). Specifically, the values $u_{0} \neq 0$ and $\lambda_{0}$ are such that

$$
G\left(u_{0}\right)-\frac{1}{2} u_{0} g\left(u_{0}\right)=0 \quad \text { and } \quad \lambda_{0}=\frac{u_{0}}{g\left(u_{0}\right)} .
$$

In this framework, one can compute the stationary traveling front $(U, V)$ by using the standard construction of a steady heteroclinic orbit for the double-well potential with wells of equal depth [17].

We conclude this section by recalling that an analytical proof of the existence of propagating fronts for the system (11) is still an open problem, in the general case $\lambda>\lambda_{\star}$, and currently under investigation. A reasonable conjecture states that, given any couple of parameters $\tau, \lambda$ with $\tau>0$ and $\lambda>\lambda_{\star}$, there is a unique propagation speed $c(\tau, \lambda)$ for the possible profile connecting the two stable steady states $(0,0)$ (on the left) and $\left(u_{+}, u_{+}\right)$(on the right).

For the limiting case $\lambda=\lambda_{\star}$, the behavior is well-described by the Zeldovich equation (more details are provided at the end of Section 3.3). Therefore, we expect that a variation of the results presented in [18] should hold, suggesting the presence of a single heteroclinic profile with a given critical (strictly positive) speed $c_{\star}$, which is exponential decreasing to 0 , together with a family of algebraically decaying profiles with speeds $c>c_{\star}$.

\section{Numerical experiments}

In this section, we perform simulations of system (11)-(12) to reproduce the special type of planar solutions to system (1) described in Section 2.4, and we discuss extensively the numerical results.

As regards the numerical strategy, we have tested different approaches and finally decided to employ an implicit-explicit finite difference algorithm. Such a choice allows adopting less expensive time steps compared to fully explicit schemes, which are instead heavily conditioned by the restrictions that stability requires. On the other hand, it avoids further computational efforts needed in the case of a fully implicit scheme, specifically dealing with large algebraic systems arising from the discretization of the non linear term inside the second equation. As a matter of fact, our numerical algorithm discretizes implicitly all the linear terms, so only an implicit treatment of the reaction function $g$ is considered. We observe that this technique does not prevent the results from keeping their quantitative and qualitative accuracy.

All numerical simulations in this article have been performed using a processor Intel(R) Core(TM) i5-3350 CPU@3.10GHz, RAM: 6.00 Gb, running under the Operating System Windows 10, 64bit.

\subsection{Discretization algorithm}

We firstly consider a spatial discretization, leading to a semi-discrete version of system (11). Denoting by $\mathrm{dx}$ the spatial mesh size, and employing a standard numerical treatment for the Laplacian, we get

$$
\left\{\begin{aligned}
\tau \frac{d u_{j}}{d t} & =v_{j}-u_{j} \\
\frac{d v_{j}}{d t} & =\frac{v_{j+1}-2 v_{j}+v_{j-1}}{\mathrm{dx}^{2}}-v_{j}+\lambda g\left(u_{j}\right)
\end{aligned}\right.
$$

where $u_{j}$ and $v_{j}$ are synthetic notations for the pointwise approximation of $u(j \mathrm{dx}, t)$ and $v(j \mathrm{dx}, t), j \in \mathbb{Z}$, in which time dependence is continuous. 
Afterwards, we introduce a semi-implicit time discretization with time step dt, by assuming that $u(j \mathrm{dx}, n \mathrm{dt})$ and $v(j \mathrm{dx}, n \mathrm{dt})$, together with their approximations $u_{j}^{n}$ and $v_{j}^{n}, n \in \mathbb{N}^{+}$, are evaluated at discrete spatio-temporal points. Therefore, the fully discrete scheme becomes

$$
\left\{\begin{aligned}
\tau \frac{u_{j}^{n+1}-u_{j}^{n}}{\mathrm{dt}} & =v_{j}^{n+1}-u_{j}^{n+1} \\
\frac{v_{j}^{n+1}-v_{j}^{n}}{\mathrm{dt}} & =\frac{v_{j+1}^{n+1}-2 v_{j}^{n+1}+v_{j-1}^{n+1}}{\mathrm{dx}^{2}}-v_{j}^{n+1}+\lambda g\left(u_{j}^{n}\right)
\end{aligned}\right.
$$

with suitable initial and boundary conditions. By means of consistency and stability arguments, it is possible to prove that the numerical method introduced above is convergent: specifically, that strategy turns out to be first order accurate in time and second order in space (see [19], for instance).

After some algebraic manipulations, we deduce a matrix equation for (16) as follows,

$$
\left(\begin{array}{cc}
\left(1+\tau^{-1} \mathrm{dt}\right) \mathbf{I} & -\tau^{-1} \mathrm{dt} \mathbf{I} \\
\mathbf{O} & (1+\mathrm{dt}) \mathbf{I}+\frac{\mathrm{dt}}{\mathrm{dx}^{2}} \mathbf{D}
\end{array}\right)\left(\begin{array}{l}
u^{n+1} \\
v^{n+1}
\end{array}\right)=\left(\begin{array}{c}
u^{n} \\
v^{n}+\lambda g\left(u^{n}\right) \mathrm{dt}
\end{array}\right)
$$

where $\mathbf{I}$ and $\mathbf{O}$ denote, respectively, the identity and the null matrice, and $\mathbf{D}=\left(-\delta_{i+1, j}+2 \delta_{i, j}-\delta_{i, j+1}\right)$ is the standard discrete Laplacian, with $\delta_{i, j}$ the Kronecker delta function of discrete variables $i$ and $j$. By taking advantage of the block-matrix structure in (17), we notice that a matrix equation for $v^{n+1}$ can be defined and separately solved, due to the low blocks independence from $u^{n+1}$. Indeed, by imposing $\mathbf{A}:=(1+\mathrm{dt}) \mathbf{I}+\frac{\mathrm{dt}}{\mathrm{dx}^{2}} \mathbf{D}$, we solve the tridiagonal linear system $\mathbf{A} v^{n+1}=v^{n}+\lambda g\left(u^{n}\right) \mathrm{dt}$ and, then, we use the function $v^{n+1}$ to update $u^{n+1}$ from the upper blocks. Such backward substitution technique avoids to operate on the whole matrix equation (17), thus allowing to save computational time.

For the experimental simulations, in order to collect information about the numerical error produced by the scheme (16), we detect a sample solution of system (11) which would play the role of an "exact" solution, by considering an extremely fine spatio-temporal mesh (precisely, we take dt $=5.0 \times 10^{-3}$ and $\mathrm{dx}=1.25 \times 10^{-2}$ ). It is important to point out how the structure of matrix $\mathbf{A}$ lends itself well to invoke the Thomas algorithm [19], which is a method of linear complexity with respect to the problem size. Actually, as it is possible to recognize by checking the results in Table 1, the computational time increases almost linearly with the size of matrix $\mathbf{A}$ (for example, by halving the mesh size, namely doubling up the matrix size, the corresponding computational time also approximately doubles up).

All numerical tests are carried out by fixing $\mathrm{dt}=5.0 \times 10^{-2}$ and $\mathrm{dx}=1.0 \times 10^{-1}$, which satisfy the so-called parabolic CFL condition [19], and the numerical solutions are compared to the sample solution by means of $L^{\infty}$ - and $L^{2}$-norm error analysis (refer to Table 1 where these values are highlighted in bold).

\subsection{Numerical evaluation of the propagation speed}

In order to provide a numerical approximation of the propagation speed, we refer to the approach originally proposed in [20], and already successfully applied to reaction-diffusion systems in [21,22].

Let us provide a brief recasting of the basic idea behind such method: given a differentiable function $\phi$ with asymptotic states $\phi_{ \pm}=\phi( \pm \infty)$, the following identity holds

$$
\int_{\mathbb{R}}[\phi(x+h)-\phi(x)] d x=h \int_{\mathbb{R}} \int_{0}^{1} \frac{d \phi}{d x}(x+\theta h) d \theta d x=h \int_{0}^{1} \int_{\mathbb{R}} \frac{d \phi}{d \eta}(\eta) d \eta d \theta=h[\phi],
$$

for some $h \in \mathbb{R}$, where $[\phi]:=\phi_{+}-\phi_{-}$, which is obtained by simply interchanging the order of integration. In particular, for an increment $h$ equal to $-c \mathrm{dt}$, the above identity provides

$$
c=\frac{1}{[\phi] \mathrm{dt}} \int_{\mathbb{R}}[\phi(x)-\phi(x-c t)] d x .
$$


Table 1. Error estimates at final time $T=100$ for three different temporal mesh sizes $\mathrm{dt}=2.5 \times 10^{-2}$ (first table), $5.0 \times 10^{-2}$ (second table) and $1.0 \times 10^{-1}$ (third table).

\begin{tabular}{c|c|c|c}
$\mathrm{dx}$ & $L^{\infty}$-error & $L^{2}$-error & CPU time \\
\hline $2.5 \times 10^{-2}$ & $1.56 \times 10^{-2}$ & $6.1 \times 10^{-3}$ & 230.63 \\
$5.0 \times 10^{-2}$ & $1.82 \times 10^{-2}$ & $7.1 \times 10^{-3}$ & 190.20 \\
$1.0 \times 10^{-1}$ & $2.34 \times 10^{-2}$ & $9.2 \times 10^{-3}$ & 53.69 \\
$2.0 \times 10^{-1}$ & $3.42 \times 10^{-2}$ & $1.34 \times 10^{-2}$ & 26.61 \\
$4.0 \times 10^{-1}$ & $5.72 \times 10^{-2}$ & $2.23 \times 10^{-2}$ & 10.61
\end{tabular}

\begin{tabular}{c|c|c|c}
$\mathrm{dx}$ & $L^{\infty}$-error & $L^{2}$-error & CPU time \\
\hline $2.5 \times 10^{-2}$ & $3.31 \times 10^{-2}$ & $1.30 \times 10^{-2}$ & 118.95 \\
$5.0 \times 10^{-2}$ & $3.57 \times 10^{-2}$ & $1.39 \times 10^{-2}$ & 56.70 \\
$\mathbf{1 . 0} \times \mathbf{1 0}^{-\mathbf{1}}$ & $\mathbf{4 . 0 9} \times \mathbf{1 0}^{-\mathbf{2}}$ & $\mathbf{1 . 5 9} \times \mathbf{1 0}^{-\mathbf{2}}$ & $\mathbf{2 7 . 9 2}$ \\
$2.0 \times 10^{-1}$ & $5.16 \times 10^{-2}$ & $2.02 \times 10^{-2}$ & 13.92 \\
$4.0 \times 10^{-1}$ & $7.47 \times 10^{-2}$ & $2.91 \times 10^{-2}$ & 5.61
\end{tabular}

\begin{tabular}{c|c|c|c}
$\mathrm{dx}$ & $L^{\infty}$-error & $L^{2}$-error & CPU time \\
\hline $2.5 \times 10^{-2}$ & $6.67 \times 10^{-2}$ & $2.60 \times 10^{-2}$ & 57.53 \\
$5.0 \times 10^{-2}$ & $6.93 \times 10^{-2}$ & $2.70 \times 10^{-2}$ & 27.63 \\
$1.0 \times 10^{-1}$ & $7.45 \times 10^{-2}$ & $2.91 \times 10^{-2}$ & 13.88 \\
$2.0 \times 10^{-1}$ & $8.52 \times 10^{-2}$ & $3.32 \times 10^{-2}$ & 6.91 \\
$4.0 \times 10^{-1}$ & $1.08 \times 10^{-1}$ & $4.20 \times 10^{-2}$ & 2.75
\end{tabular}

Assuming that $\phi_{j}^{n+1}$ is an approximation - in the sense of a propagating front - of $\phi\left(x_{j}-c t^{n}\right)$, with $x_{j}=j \mathrm{dx}$ and $t^{n}=n \mathrm{dt}$, we numerically compute the integral in (18) by means of the midpoint algorithm and we deduce the LeVeque-Yee formula for the discrete wave speed, namely

$$
c_{L Y}^{n}:=\frac{\sum_{j}\left(\phi_{j}^{n}-\phi_{j}^{n+1}\right) \mathrm{dx}}{[\phi] \mathrm{dt}} .
$$

Such approximation is indeed exact whenever $\phi_{j}^{n}$ is related to a traveling wave solution $\phi$ with constant velocity $c$ and asymptotic states $\phi_{ \pm}$. In general, the value $c_{L Y}^{n}$ in (19) can be regarded as a space-averaged propagation speed, which stabilizes to $c$ when $\phi_{j}^{n}$ converges to the given traveling profile $\phi$.

Because system (11) has two dynamical variables $u$ and $v$, the respective speed values can be computed through the LeVeque-Yee formula (19) as

$$
c_{u, L Y}^{n}:=\frac{\sum_{j}\left(u_{j}^{n}-u_{j}^{n+1}\right) \mathrm{dx}}{[u] \mathrm{dt}} \quad \text { and } \quad c_{v, L Y}^{n}:=\frac{\sum_{j}\left(v_{j}^{n}-v_{j}^{n+1}\right) \mathrm{dx}}{[v] \mathrm{dt}},
$$

thus furnishing two (possibly distinct) values $c_{u, L Y}^{n}$ and $c_{v, L Y}^{n}$.

There are two possible approaches to validate the approximation (20) for experimental simulations, either by comparison with a variation of the Rankine-Hugoniot relation for reaction-diffusion equations [23], or rather by putting together with the identity (15). Note that, in both the last methods, the dynamics of system (11) is explicitly taken into account to evaluate the propagation speed; differently, for establishing the formula (20), the dynamics of the couple $(u, v)$ issued from the PDE model is never used. We regard at this property as an irreproachable advantage of the LeVeque-Yee formula.

More precisely, we call a variation of the Rankine-Hugoniot relation simply the result of a separate integration of the balance laws constituting the one-dimensional system (11). Assuming the special type of solutions $u(x, t)=U(\xi)$ and $v(x, t)=V(\xi)$, with $\xi=x-c t$, by integrating with respect to $x$ the first and second equation of system (13), we obtain

$$
c_{u, R H}=\frac{1}{\tau[u]} \int_{\mathbb{R}}(U-V) d x \quad \text { and } \quad c_{v, R H}=\frac{1}{[v]} \int_{\mathbb{R}}[V-\lambda g(U)] d x,
$$


where $[u]=U_{+}-U_{-}$and $[v]=V_{+}-V_{-}$with the asymptotic states from Definition 2.1. A standard discretization of the above integrals produces

$$
c_{u, R H}^{n}=\frac{\sum_{j}\left(u_{j}^{n}-v_{j}^{n}\right) \mathrm{dx}}{\tau[u]}=\frac{\sum_{j}\left(u_{j}^{n}-u_{j}^{n+1}\right) \mathrm{dx}}{[u] \mathrm{dt}}=c_{u, L Y}^{n},
$$

where we have used the first equation of (16). Analogously, for the second equation it holds

$$
c_{v, R H}^{n}=\frac{\sum_{j}\left[v_{j}^{n}-\lambda g\left(u_{j}^{n}\right)\right] \mathrm{dx}}{[v]}=(1+\mathrm{dt}) \frac{\sum_{j}\left(v_{j}^{n}-v_{j}^{n+1}\right) \mathrm{dx}}{[v] \mathrm{dt}}=(1+\mathrm{dt}) c_{v, L Y}^{n},
$$

thanks to the property of null summation of the discrete Laplacian in (16).

This computation shows that - apart from a multiplying factor of order $\mathrm{dt}$ - the variation of the RankineHugoniot relation and the LeVeque-Yee formula are equivalent and, therefore, they cannot be considered as a reliable test for establishing the validity of each other.

On the other hand, comparing the formula (20) with the exact identity (15) is more favorable, as a consequence of the fact that an independent procedure is required for obtaining a discrete version of (15). Here, we rely on the results provided in Figure 4 to support the validity of the LeVeque-Yee formula (20).
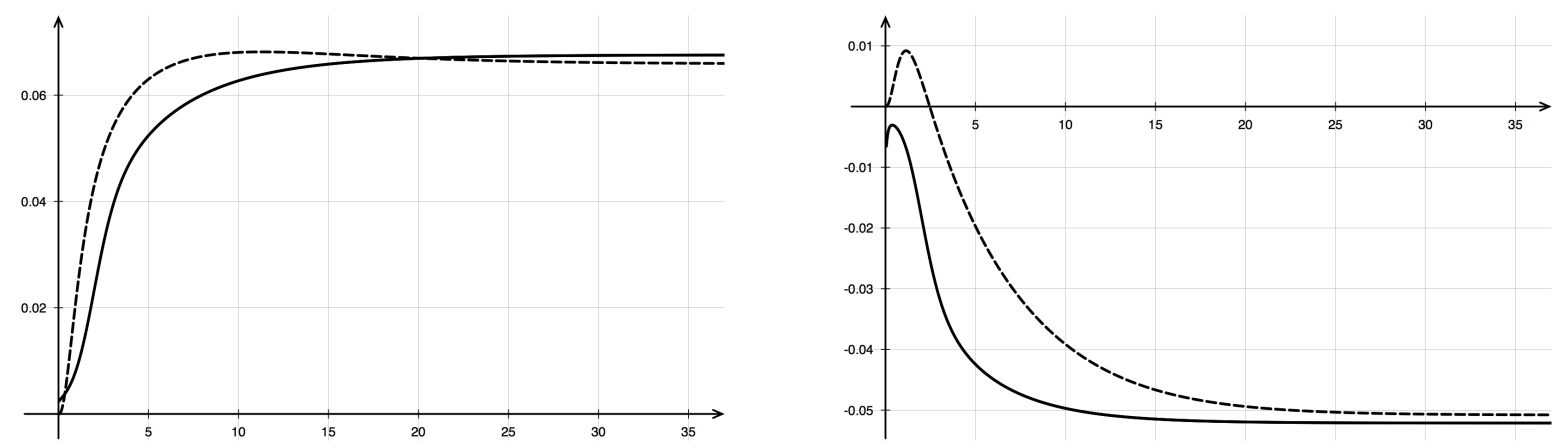

Figure 4. Comparison between the function $t \mapsto c(t)$ for $t \in(0,35)$ as given by the exact identity (15) (continuous) and the estimation by the LeVeque-Yee formula (20) (dashed) relative to the values $\lambda=2.1$ (left plot) and $\lambda=2.25$ (right plot).

The errors exhibited in the simulations about the (constant) asymptotic speed values have actually a size of order $10^{-3}$ and, hence, these numerical results are widely acceptable. Similar experimental errors are also shared by different ranges of the parameter $\lambda$ and are omitted.

\subsection{Computational results}

In the limiting case $\tau=0$, the model (11) reduces to a standard parabolic reaction-diffusion equation for the mesenchymal phenotype, whose dynamical behavior is well-known (see [17] and references therein).

As already discussed in Section 2, no invasion is possible for $0<\lambda<\lambda_{\star}$ and a complete Mesenchymalto-Epithelial Transition (MET) represented by the regression to the steady state $(0,0)$ is always the final fate of the solution $(u, v)$. Then, increasing $\lambda$ and trespassing the first threshold $\lambda_{\star}$, but staying below the second threshold $\lambda_{0}$, translates into the presence of traveling waves with positive speed, thus corresponding again to the case of MET regression. Finally, for choices of $\lambda>\lambda_{0}$, the traveling wave passes from positive to negative values of the speed, corresponding to the case of a possible invasive regime, which is typical of an Epithelial-to-Mesenchymal Transition (EMT).

The general case $\tau>0$ follows the same qualitative analysis with respect to the parameter $\lambda$. In particular, for the reaction function (7) with $p=2$, the thresholds $\lambda_{\star}$ and $\lambda_{0}$ can be explicitly computed and are given by

$$
0<\lambda_{\star}=2.0<\lambda_{0}=2.175063 .
$$

For $\lambda<\lambda_{\star}$, the situation is straightforward, since any positive initial datum generates a solution that converges to $(0,0)$ as $t \rightarrow+\infty$ with exponential rate. Then, we concentrate on the regime $\lambda \geq \lambda_{\star}$. 
The numerical results reported below describe only the profile for the component $u$, the profile of $v$ being qualitatively very similar. Here, we limit the presentation to the evolution dynamics exhibited by the Riemann problem, but we can guarantee that also much more oscillating initial data show the same kind of large-time behavior. In addition, we provide different graphical representations of numerical solutions with the same scale in the vertical axis, so that the height and width of the front profiles can be compared between different simulations.

The discussion of the critical case $\lambda=\lambda_{\star}=2.0$ is postponed to the final part of this section.

$\lambda=2.1 \in\left(\lambda_{\star}, \lambda_{0}\right)$ - For this choice of the parameter $\lambda$, numerical evidence of the existence of a traveling front is obtained. Moreover, being the stable state $u_{+}(\lambda)$ closer to the critical state corresponding to the threshold value $\lambda_{\star}$, the solution exhibits a regressive (MET) behavior, namely the front travels toward the right-hand side with positive speed (refer to Figure 5).

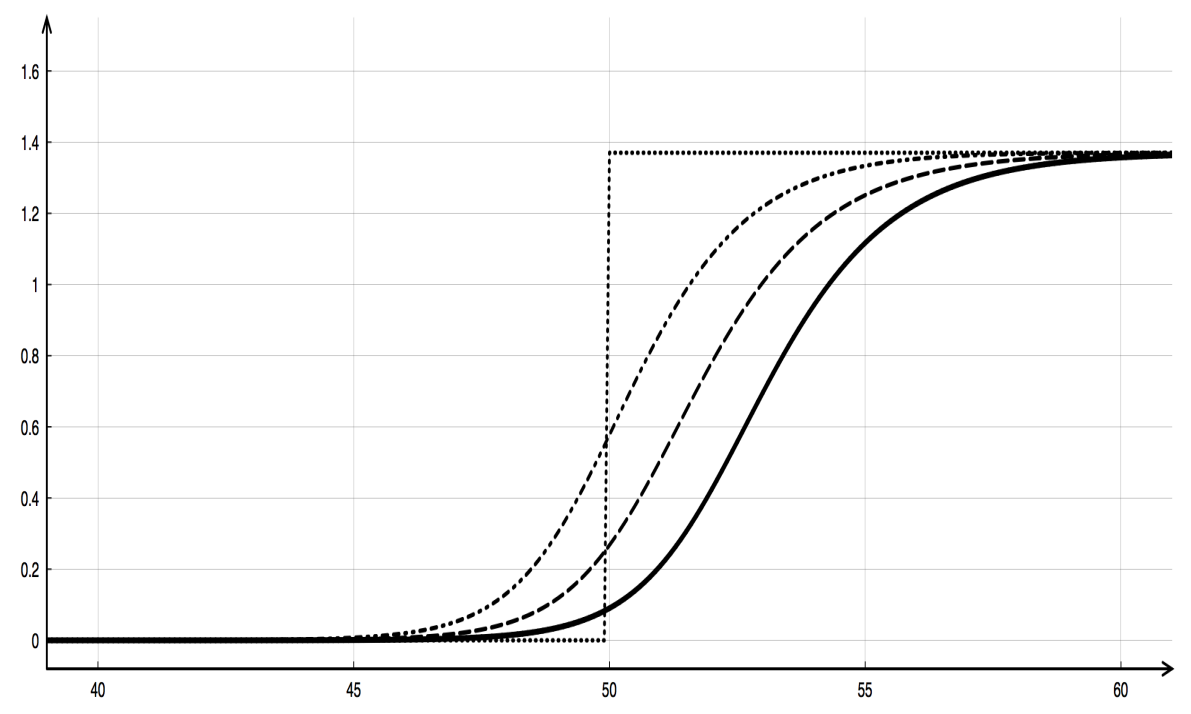

Figure 5. Regressive regime (MET) for $\lambda=2.1$. Numerical simulation of the traveling wave solution to the Riemann problem at times $t=0$ (dotted), $t=10$ (dash-dotted), $t=30$ (dashed) and $t=50$ (continuous).

$\lambda=\lambda_{0} \approx 2.175063$ - Since the two wells of the potential function (9) have the same depth for this value of $\lambda$ (refer to Figure 3), system (11) possesses a stationary solution with the required asymptotic behavior for $\tau=0$. In particular, the dynamics is independent from the relaxation parameter $\tau$ and the existence of a traveling wave in the regime $\tau>0$ is a straightforward consequence of the observation that the fronts are actually steady states. For a short time-scale, the solution to the Riemann problem converges to a smoothed version of the jump from $u=0$ to $u=u_{+}\left(\lambda_{0}\right)$. Thus, for the sake of shortness, we do not present any numerical simulation for such a simple dynamics.

$\lambda=2.25>\lambda_{0}-$ Again, numerical evidence of the existence of propagating fronts emerges as the long-time behavior of the solution to the Riemann problem (refer to Figure 6). The traveling wave has now a positive speed, so that we are in a situation for which invasion (EMT) is possible, at least for Riemann initial data. For more general initial data, a sort of competition between different parts of the solution starts playing a crucial role in the determination of the large-time behavior.

As far as $\lambda$ increases, the numerically computed speed of the propagating front increases in absolute value and, thus, invasive EMT regimes are more and more probable (see Section 3.4).

Finally, we come back to the case of the threshold value $\lambda_{\star}$.

$\lambda=\lambda_{\star}=2.0-$ As previously observed, the behavior of the solutions in this limiting case should be described analogously to what is done in [18] and its descendants for the equation

$$
\partial_{t} u=\partial_{x x} u+u^{2}(1-u)
$$




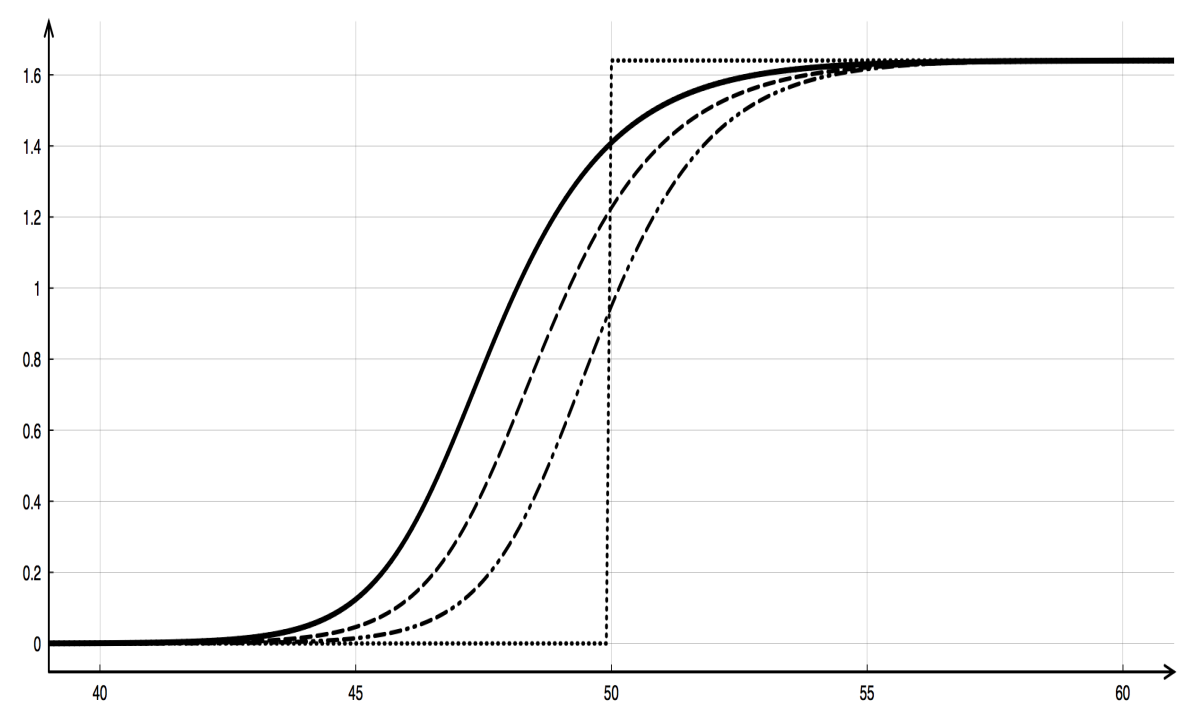

Figure 6. Invasive regime (EMT) for $\lambda=2.25$. Numerical simulation of the traveling wave solution to the Riemann problem at times $t=0$ (dotted), $t=10$ (dash-dotted), $t=30$ (dashed) and $t=50$ (continuous).

whose characteristic is the presence of a multiple (second-order) zero at 0 . We note that equation (21) is sometimes referred to in the literature as the Zeldovich equation and it typically arises in the description combustion phenomena (see [24], for instance).

In this framework, there exist infinitely many traveling wave solutions (up to translations), with one profile for any given speed greater than or equal to some positive threshold value $c_{\star}$ (which has an explicit representation exactly when the specific equation (21) is considered). The distinguishing feature of the profile $U_{\star}$ associated to the speed $c_{\star}$ is that it is the unique profile with an exponential decay to both the asymptotic states. On the contrary, whenever $c>c_{\star}$, the decay to the unstable state has merely an algebraic rate. We conjecture the same to be true also for the bi-dimensional system (11), although at the moment we are not able to give an analytical proof of such a statement.

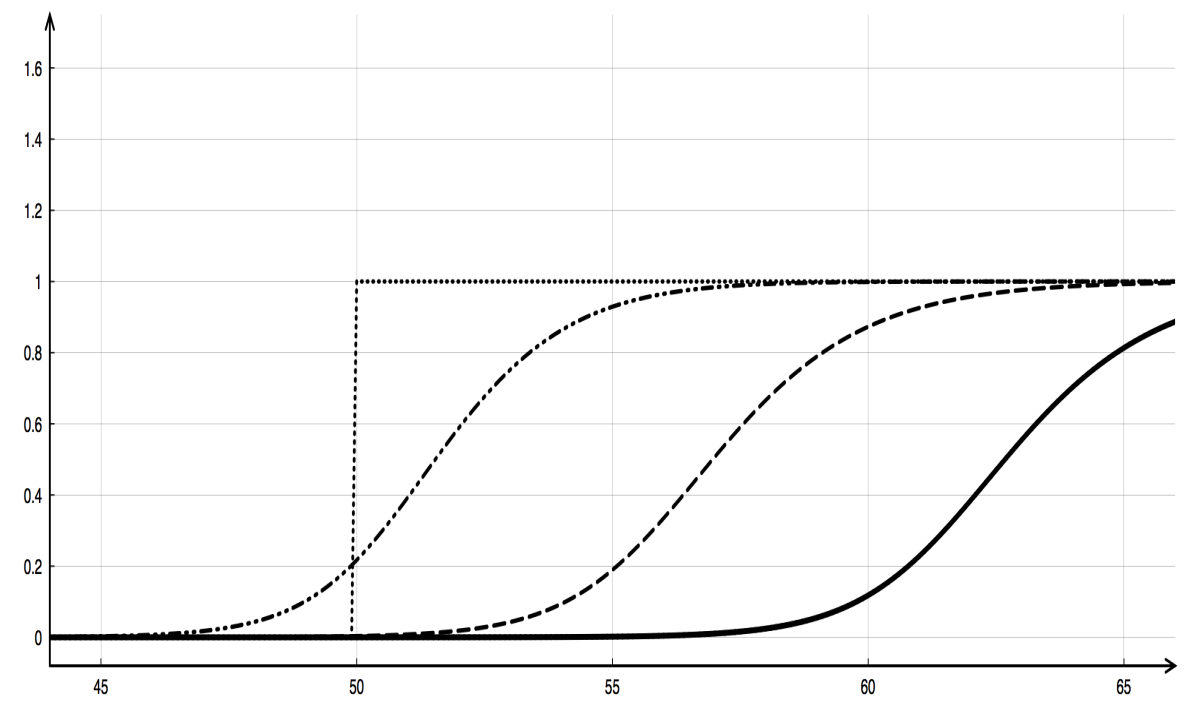

Figure 7. Critical regime for $\lambda=\lambda_{\star}=2.0$. Numerical simulation of the traveling wave solution to the Riemann problem at times $t=0$ (dotted), $t=10$ (dash-dotted), $t=30$ (dashed) and $t=50$ (continuous).

Here, we limit the presentation to numerical results of the large-time behavior determined by Riemann initial data, with asymptotic states given by $(0,0)$ and $\left(u_{+}\left(\lambda_{\star}\right), u_{+}\left(\lambda_{\star}\right)\right)$. The emergence of a traveling 
wave solution is apparent, propagating to the right-hand side with some speed $c_{\star}>0$ (refer to Figure 7 ).

\subsection{Sensitivity analysis with respect to $\tau$ and $\lambda$}

We finally turn our attention to the sensitivity of the numerical solutions to system (11) with respect to the parameters $\tau$ and $\lambda$, which is measured by considering as principal unknown the speed of propagation of the traveling fronts as evaluated by the LeVeque-Yee formula (20).

Relaxation time $\tau$. The dependence from the relaxation parameter $\tau$ is very smooth and it does not manifest any special feature from a numerical point of view (refer to Figure 8).

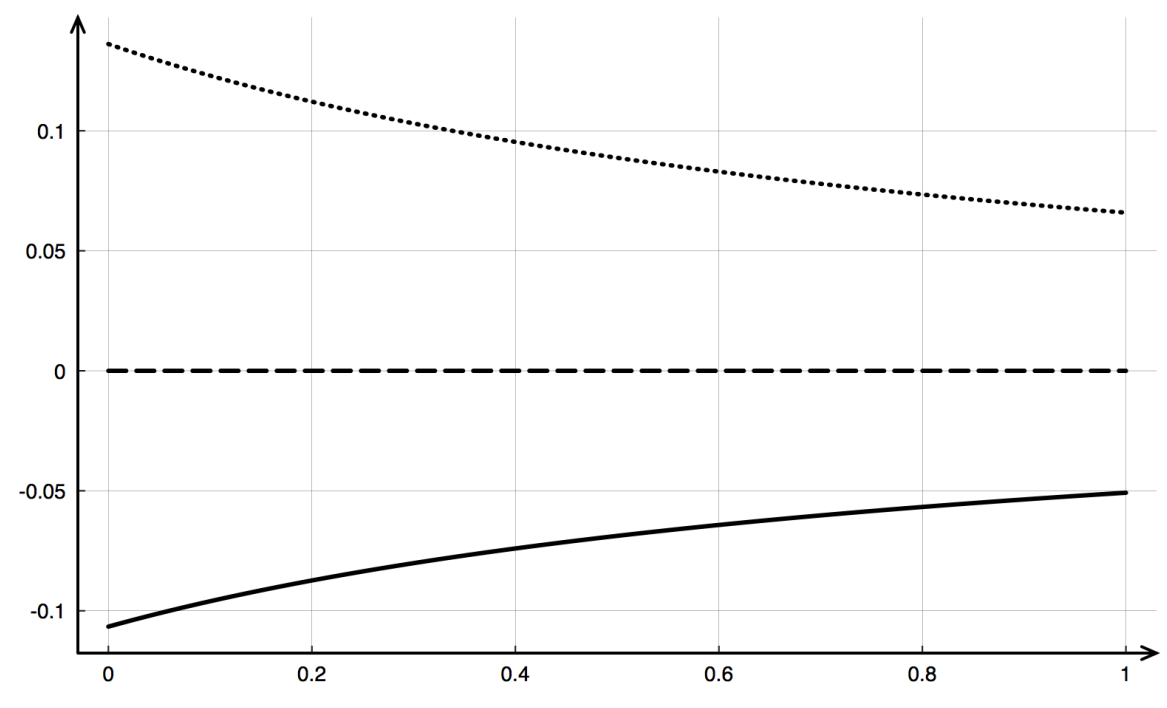

Figure 8. Graphs of $c(\cdot, \lambda)$ as a function of the relaxation parameter $\tau \in[0,1]$ for $\lambda=2.1$ (dots), $\lambda=\lambda_{0}=2.175063$ (dashed line) and $\lambda=2.25$ (continuous), computed at the final time $T=100$.

Monotonicity of the propagation speed with respect to $\tau$ can be conjectured as a computational evidence, at least in the range of values under consideration. For $\tau>0$ and $\lambda \in\left(\lambda_{\star}, \lambda_{0}\right)$, the graph of the function is monotone decreasing, while the monotonicity is reversed in the complementary regime $\lambda>\lambda_{0}$. The two regimes are separated by the threshold value $\lambda_{0}=2.175063$, which indeed corresponds to the emergence of a stationary solution. This translates into the fact that both regression (MET) and invasion (EMT) are slowed down when $\tau$ increases, and that modification is actually relevant, since the regression/invasion speed changes by a percentage greater than $50 \%$ when $\tau$ passes from 0 to 1 . Hence, the relaxation time $\tau$ has a (smoothly) distributed delay-type impact on the dynamics, and this feature has to be taken into account while building more quantitative models.

Invasiveness parameter $\lambda$. As expected, the dependence from the parameter $\lambda$ is more interesting, the results exhibiting a strong variation in the vicinity of the threshold value $\lambda_{\star}$ (refer to Figure 9).

As a matter of fact, the function $c(\tau, \cdot)$ is monotonically decreasing, that reproduces - at least numerically - the experimental observation of propagating fronts expecting to become more and more invasive as $\lambda$ increases (namely, the preeminent motion is toward the left for our choice of the Riemann data). In particular, the graph of the variation function $\partial_{\lambda} c(\tau, \cdot)$ suggests that $\partial_{\lambda} c(\tau, \lambda) \rightarrow-\infty$ as $\lambda \rightarrow \lambda_{\star}$.

We conclude this section by recalling that, as a consequence of the convexity/concavity assumptions on the reaction function $g$, for any $\lambda>\lambda_{\star}$ the system (11) possesses two stable steady states $(0,0)$ and $\left(u_{+}(\lambda), v_{+}(\lambda)\right)$, and therefore it is expected to support a propagating front connecting these asymptotic values (see Section 2.3). It is particularly interesting to remark how the simple PDE model (11) integrates the typical property of propagating fronts of being/not being invasive merely thanks to the use of two control parameters $\tau$ and $\lambda$. Moreover, we have determined explicit transition thresholds $\lambda_{\star}$ and $\lambda_{0}$ which separate regression regimes (MET) from invasive ones (EMT), and these parameters are indeed 

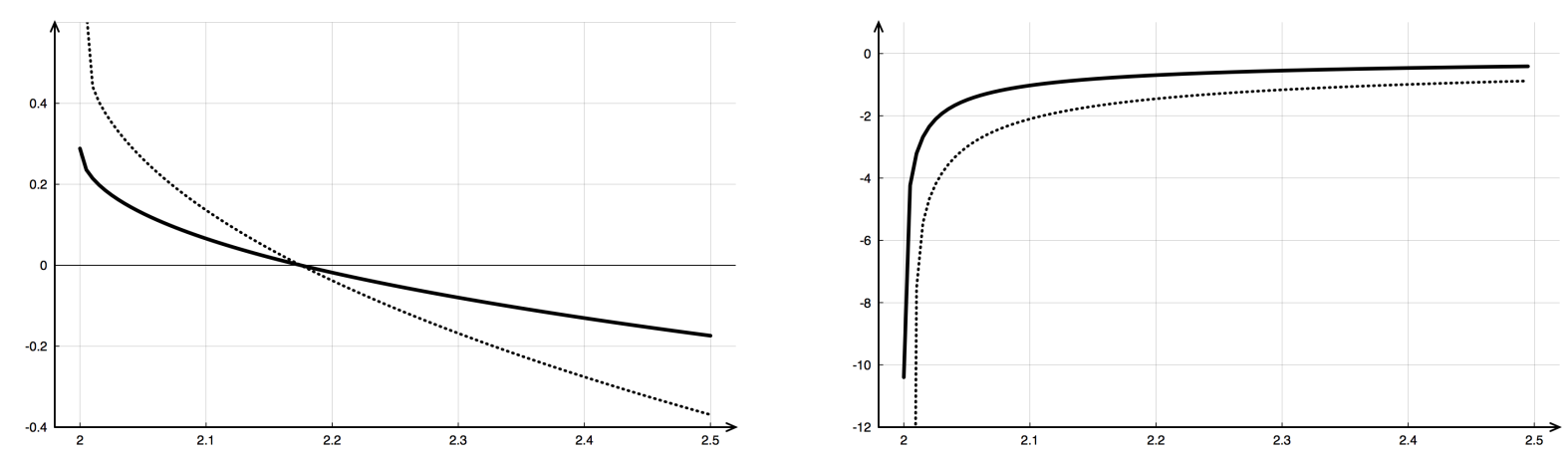

Figure 9. Graphs of $c(\tau, \cdot)$ (left plot) and its variation $\partial_{\lambda} c(\tau, \cdot)$ (right plot) as functions of the invasiveness parameter $\lambda \in[2,2.5]$ for $\tau=0$ (dots) and $\tau=1$ (continuos), computed at the final time $T=100$.

independent from the value of the relaxation time $\tau$. Also, in the limiting case $\tau=0$, system (11) reduces to a standard scalar reaction-diffusion equation and, thus, a possible strategy for obtaining a complete and rigorous proof of the existence of traveling waves could be based on singular perturbation techniques (with respect to the parameter $\tau$ ).

\section{Acknowledgements}

This article has been greatly motivated by fruitful discussions with the researchers of the Systems Biology Group Lab - www.sbglab.org - settled at the Department of Experimental Medicine, Sapienza University of Rome (Italy) and, in particular, with M. Bizzarri and S. Dinicola.

The authors are also grateful to A. Corli for constructive conversations about the Zeldovich equation reported at the end of Section 3.3.

\section{REFERENCES}

1. D. Yao, C. Dai and S. Peng, Mechanism of the Mesenchymal-Epithelial Transition and its relationship with metastatic tumor formation, Molecular Cancer Research, vol. 9, no. 12, pp. 1608-1620, 2011.

2. J.P. Thiery and J.P. Sleeman, Complex networks orchestrate epithelial-mesenchymal transitions, $N a$ ture Reviews Molecular Cell Biology, vol. 7, no. 2, pp. 131-142, 2006.

3. P.C. Davies, L. Demetrius and J.A. Tuszynki, Cancer as dynamical phase transition, Theoretical Biology and Medical Modelling, vol. 8, pp. 1-30, 2011.

4. M. Mojtahedi, A. Skupin, J. Zhou, I.G. Castaño, R.Y.Y. Leong-Quong, H. Chang, K. Trachana, A. Giuliani and S. Huang, Cell fate decision as high-dimensional critical state, PLOS Biology, vol. 14, no. 12 , p. e2000640, 2016.

5. J. Xu, S. Lamouille and R. Derynck, TGF- $\beta$-induced epithelial to mesenchymal transition, Cell Research, vol. 19, no. 2, pp. 156-172, 2009.

6. M. Bertolaso, Philosophy of cancer. A dynamic and relational view. History, Philosophy and Theory of the Life Sciences 18, Springer Science+Business Media Dordrecht, Springer Netherlands, 2016.

7. C. Simeoni, S. Dinicola, A. Cucina, C. Mascia and M. Bizzarri, Systems Biology approach and Mathematical Modeling for analyzing phase-space switch during Epithelial-Mesenchymal Transition, in Systems Biology (M. Bizzarri, ed.), Methods in Molecular Biology 1702, pp. 95-123, Springer Protocols+Business Media LLC, 2018.

8. M. Bizzarri, A. Cucina, F. Conti and F. D'Anselmi, Beyond the oncogene paradigm: understanding complexity in carcinogenesis, Acta Biotheoretica, vol. 56, no. 3, pp. 173-196, 2008. 
9. G. Barrière, P. Fici, G. Gallerani, F. Fabbri and M. Rigaud, Epithelial Mesenchymal Transition: a double-edged sword, Clinical and Translational Medicine, vol. 4, no. 14, pp. 1-6, 2015.

10. M.W. Green and B.D. Sleeman, On FitzHugh's nerve axon equations, Journal of Mathematical Biology, vol. 1, pp. 153-163, 1974.

11. M.A. Jones, B. Song and D.M. Thomas, Controling wound healing through debridement, Mathematical and Computer Modelling, vol. 40, pp. 1057-1064, 2004.

12. R. Gesztelyi, J. Zsuga, A. Kemeny-Beke, B. Varga, B. Juhasz and A. Tosaki, The Hill equation and the origin of quantitative pharmacology, Archive for History of Exact Sciences, vol. 66, pp. 427-438, 2012.

13. J. Monod, J. Wyman and J.P. Changeux, On the nature of allosteric transitions: a plausible model, Journal of Molecular Biology, vol. 12, pp. 88-118, 1965.

14. J.N. Weiss, The Hill equation revisited: uses and misuses, The FASEB Journal, vol. 11, no. 11, pp. 835-841, 1997.

15. R.I. Masel, Principles of adsorption and reaction on solid surfaces, Wiley Series in Chemical Engineering 3, John Wiley \& Sons, 1996.

16. C.S. Holling, Some characteristics of simple types of predation and parasitism, The Canadian Entomologist, vol. 91, no. 7, pp. 385-398, 1959.

17. P.C. Fife and J.B. McLeod, The approach of solutions of nonlinear diffusion equations to travelling front solutions, Archive for Rational Mechanics and Analysis, vol. 65, pp. 335-361, 1977.

18. J.A. Sherratt and B.P. Marchant, Algebraic decay and variable speeds in wavefront solutions of a scalar reaction-diffusion equation, IMA Journal of Applied Mathematics, vol. 56, pp. 289-302, 1996.

19. A. Quarteroni, Numerical models for differential problems. Third edition. Modeling, Simulation and Applications 16, Springer, Cham, 2017.

20. R.J. LeVeque and H.C. Yee, A study of numerical methods for hyperbolic conservation laws with stiff source terms, Journal of Computational Physics, vol. 86, no. 1, pp. 187-210, 1990.

21. C. Lattanzio, C. Mascia, R.G. Plaza and C. Simeoni, Kinetic schemes for assessing stability of traveling fronts for the Allen-Cahn equation with relaxation, Applied Numerical Mathematics, vol. 141, pp. 234$247,2019$.

22. P. Moschetta and C. Simeoni, Numerical investigation of the Gatenby-Gawlinski model for acidmediated tumour invasion, Rendiconti di Matematica e delle sue Applicazioni, pp. 1-31, online first, 2019 .

23. R.J. LeVeque, Finite difference methods for ordinary and partial differential equations. Steady-state and time-dependent problems. Society for Industrial and Applied Mathematics (SIAM), Philadelphia, PA, 2007.

24. B.H. Gilding and R. Kersner, Travelling waves in nonlinear diffusion-convection reaction. Progress in Nonlinear Differential Equations and Their Applications 60, Springer Basel AG, 2004. 